\title{
Los objetos de aprendizaje del Portal Educ.ar: inclusión de tecnologías digitales en materiales didácticos de lengua y literatura'
}

\author{
The Learning Objects of the Educ.ar Portal:The inclusion of Digital \\ Technologies in Language and Literature Teaching Materials
}

\author{
ALEJO EZEQUIEL GONZÁLEZ LÓPEZ LEDESMA \\ Universidad Nacional de General Sarmiento - CONICET \\ Argentina \\ alejogll@hotmail.com
}

(Recibido: 29-10-2015; aceptado: 19-03-2016)

Resumen. En este estudio se presentan los primeros avances de una investigación de tesis de maestría cuyo tema es la inclusión de tecnologías digitales en los materiales didácticos de Lengua y Literatura del Portal Educ.ar dedicados a la enseñanza de la escritura. A lo largo de este trabajo, expondremos nuestro marco teórico, que incorpora tanto las discusiones en torno a los materiales didácticos que incluyen tecnologías digitales como el enfoque sobre la enseñanza de la escritura que guía nuestro análisis. Luego, detallaremos la metodología utilizada y expondremos los resultados de nuestro análisis a partir de una serie de ejemplos representativos de la muestra seleccionada. De este modo, buscaremos dar cuenta de la forma en que un enfoque didáctico-disciplinar puede arrojar luz sobre las posibilidades que ofrece la inclusión de tecnologías digitales para la enseñanza de la escritura en el marco de las propuestas de los materiales didácticos.

Palabras clave: Escritura; lengua; literatura; tecnología educacional; material didáctico.
Abstract. This study aims to introduce the first conclusions reached in a master's thesis study on the inclusion of digital technologies in teaching materials belonging to the Portal Educ.ar and dedicated to writing instruction. Throughout this paper, we will explain our theoretical framework, which articulates the discussions on teaching materials with our particular view on writing instruction. Then we will introduce the methodological framwork used along our study and, using various examples, we will present the results analysis. By doing this, we will be able to understand the ways in which a didactic and disciplinary view can shed light on the possibilities offered by the inclusion of digital technologies in teaching materiales and writing instruction.

Keywords: Written language; language; literature; educational technology; teaching materials.

\footnotetext{
${ }^{1}$ Para citar este artículo: González López Ledesma, Alejo Ezequiel (2016). Los objetos de aprendizaje del portal Educ.ar: inclusión de tecnologías digitales en materiales didácticos de lengua y literatura. Alabe 13. [www.revistaalabe.com] DOI: 10.15645/Alabe2016.13.5
} 


\section{Introducción}

El siguiente trabajo presenta los primeros avances de una tesis de maestría cuyo objetivo es contribuir a la reflexión sobre la enseñanza de la escritura en la disciplina Lengua y Literatura ${ }^{2}$, a través del análisis de las características que adopta la inclusión de tecnologías digitales en los materiales didácticos del Portal Educ.ar del Ministerio de Educación de la Nación. En este primer avance, se presentarán los resultados preliminares -y provisorios- del proceso de análisis y codificación de datos que ha sido realizado luego de haber llevado adelante un primer relevamiento y una selección de los materiales didácticos que conforman el corpus de la investigación.

Esta investigación parte de la premisa de que, por el cambio cultural que representa, la inclusión masiva de nuevas tecnologías en la escuela nos interpela a revisar y expandir los marcos interpretativos de las prácticas de enseñanza (Maggio, Lion \& Sarlé, 2012). En el marco de este nuevo escenario, resulta fundamental desarrollar investigaciones que apunten a comprender la forma en que las tecnologías, que implican "una reestructuración de lo que entendemos por conocimiento, de las fuentes y los criterios de verdad, y de los sujetos autorizados y reconocidos como productores de conocimiento" (Dussel, 2011:16), entran en diálogo con otra cultura, aquella de la escuela, cuyos hábitos, tiempos y valores adscriben a otro tipo de organización del saber, más jerárquica y centralizada.

A fin de repensar esta problemática educativa en función del objeto que configuran los materiales didácticos de LyL, se explicitará el marco teórico desde el cual abordamos, por un lado, los materiales didácticos que incorporan tecnologías digitales, y por otro, los libros de textos de LyL, que configuran antecedentes directos del objeto de estudio en cuestión. Luego, introduciremos la metodología utilizada para llevar adelante el análisis de los Objetos de Aprendizaje ${ }^{3}$ y expondremos los resultados provisorios de la investigación. Por último, introduciremos algunas conclusiones provisorias que dan cuenta de las líneas de análisis que se abren a partir del estudio de esta primera muestra de materiales.

\footnotetext{
${ }^{2}$ A partir de ahora, LyL.

${ }^{3}$ A partir de ahora, OA.
} 


\section{Marco teórico}

\subsection{Los Objetos de Aprendizaje}

A lo largo de las últimas décadas, se han desarrollado en la Argentina diversos estudios dedicados a investigar las características que presentan los materiales didácticos en relación con las mediaciones tecnológicas que los atraviesan. Sin desconocer los valiosos aportes de otros trabajos en esta área, hemos realizado un recorte conformado particularmente por aquellos estudios dedicados al nivel de enseñanza superior en el contexto de los entornos virtuales de aprendizaje (De Angelis, Gergich \& Imperatore, 2010; Gergich, Imperatore \& Schneider, 2012). Esto se debe a que estas investigaciones han logrado configurar un cuerpo de problemas en torno a las dimensiones tanto didácticas como tecnológicas de los materiales que resulta útil a los fines de presentar con mayor detalle el enfoque específico que ofrecemos en nuestra investigación.

Para comenzar, las investigaciones en cuestión describen las características de los materiales didácticos a través de la lente de la historia de transformaciones atravesadas desde las últimas décadas del siglo XX hasta la actualidad. Ese proceso de historización expone las mutaciones que los materiales didácticos han sufrido en su relación dialéctica con los cambios tecnológicos. Más allá de las diferencias entre sus objetos de estudio, estos trabajos coinciden en que el estado actual en el que se encuentran los materiales didácticos imposibilita pensarlos autónomamente, por fuera de las complejas variables que configuran las propuestas que sirven de marco para interpretar y también diseñar esos materiales. La metáfora que de manera más ajustada condensa esta idea es la del estallido (Gergich et al., 2012).

Concebir los materiales en términos de un estallido nos habilita a comprender una transformación dada por un pasaje que va desde la autonomía, la textualidad y la legitimidad garantizada por la autoría, la autorreferencialidad y la mirada del material como medio o instrumento, propios de una etapa inicial, a otro momento, más actual, atravesado por una concepción que contempla a los materiales como procesos complejos o mediaciones, como objetos que son fruto de la articulación con las restantes variables contextuales que intervienen en las propuestas educativas -tales como los entornos virtuales, las estrategias de enseñanza y los participantes de los cursos- y como objetos en permanente cambio y desarrollo, gracias a su carácter polifónico y abierto.

Paralelamente, el concepto de estallido resulta fundamental para comprender cabalmente el desarrollo conceptual del enfoque que adoptaremos más adelante. La multiplicación de variables que involucra tanto para el análisis de los materiales como para su diseño da como resultado un escenario que, tal como lo entendemos, se encuentra atravesado por la unicidad: a través de la lente del estallido, cada material supone una síntesis de variables tan diversas como experiencias educativas existan; cada curso está marcado por el signo de lo irrepetible. Cuando los elementos contextuales cobran relevancia, entonces pasa a ser perceptible el modo en que en el seno del diseño de cada material varían los alumnos, docentes y tutores y, por tanto, los circuitos comunicativos, las estrategias 
didácticas y los entornos de aprendizaje.

Ahora bien, la metáfora del estallido nos devuelve como respuesta una incógnita cuando intentamos aplicarla a otros materiales cuyo diseño ha sido guiado por criterios menos contextualizados, únicos o irrepetibles. Tal es el caso de los materiales didácticos del Portal Educ.ar, objeto de estudio de esta investigación.

Fundado en el año 2000 y relanzado en el año 2003 bajo el gobierno de Néstor Kirchner, Educ.ar constituye hoy el portal educativo del Ministerio de Educación de la Argentina. Ofrece una plataforma de formación a distancia, recursos y contenidos didácticos, entre otros servicios del mundo digital, con el propósito de incorporar las nuevas tecnologías de la información y la comunicación a la enseñanza. Desde 2010, el Portal participa del programa Conectar Igualdad, para el cual, hasta la actualidad, ha producido alrededor de veinte mil OA destinados a docentes y alumnos.

La denominación específica de OA que el mismo portal les adjudica a los veinte mil materiales didácticos no es casual. En efecto, se condice con las características que pueden observarse en los materiales del Portal y que Prendes Espinosa, Martínez Sánchez y Gutiérrez Porlán (2008) sintetizan en su definición: los OA son unidades digitales de información, contenidos autónomos, con las características de interactividad, independencia, la posibilidad de combinarse en unidades u objetos de mayor tamaño, la capacidad de adaptación a los usuarios y de ser reutilizados en contextos de enseñanza y aprendizaje variados. La definición se completa con otra de las características constitutivas de los OA, aquella que les otorga su lugar en el Portal Educ.ar: los OA se encuentran en repositorios o acervos, que son colecciones de OA asociados a información o metadatos. Estos metadatos, al mismo tiempo que habilitan la catalogación digital de los OA, permiten su búsqueda y su reutilización.

Las críticas que se han formulado contra los OA y sus repositorios-categoría, esta última, aplicable sin inconvenientes al Portal Educ.ar- ponen en cuestión su rol en los procesos de enseñanza y aprendizaje. A diferencia de lo que sucede con las propuestas desarrolladas en el marco de los entornos virtuales de aprendizaje, los OA constituyen materiales didácticos con un mayor grado de descontextualización, ya que no han sido diseñados para los contextos en lo que son utilizados y reutilizados. A su vez, según sus detractores, alientan prácticas de reproducción, ya que, al usarlos, los docentes pasan más tiempo realizando búsquedas de OA en catálogos que produciendo materiales vinculados a su contexto de trabajo específico. De aquí que, según Sabulsky (2009), la estandarización y la omisión de variables contextuales que trae aparejado el uso de OA los ubica por fuera de la categoría de materiales didácticos y los reposiciona entre los recursos para la enseñanza, definidos estos últimos únicamente por su uso en contextos de enseñanza, al margen de que hayan sido diseñados para cumplir tal función. Desde esta perspectiva, entonces, podríamos decir, a modo de ejemplo, que una foto o una piedra son recursos didácticos y un OA lo es de igual forma.

En este trabajo, asumimos, por un lado, que los $\mathrm{OA}$ no constituyen materiales didácticos en el sentido que han planteado los trabajos desarrollados en el marco de los 
entornos virtuales de aprendizaje. También coincidimos en que el diseño de los OA no contempla las variables que hacen a un material diseñado para una experiencia educativa específica y entendemos que resulta necesario discutir los procesos de estandarización en la creación de materiales. No obstante, por otro lado, resulta necesario señalar que los OA del Portal Educ.ar, objeto de nuestro análisis, no constituyen tampoco recursos educativos. A diferencia de las fotos que podemos encontrar en un diario o las canciones de un disco compacto, los OA que estudiamos están atravesados en sus diseños por configuraciones del orden de lo didáctico. Por eso mismo, asumimos aquí la caracterización que plantea Área Moreira (2004) -que también es retomada por Imperatore (2009: 358)-, en el marco de la cual la dimensión simbólica y didáctica resulta constitutiva de lo que se entiende por material didáctico: “además de su parte física, (el material) codifica un sistema de símbolos que estructura mensajes con significado y que lleva inscriptas las huellas de las mediaciones culturales y didácticas que se activarán con las estrategias de enseñanza". Siguiendo esta línea, entonces, nos abocaremos aquí a investigar específicamente las mediaciones culturales y didácticas que atraviesan la configuración de los OA del Portal Educ.ar ${ }^{4}$.

\subsection{Didáctica de la Lenguay la Literatura: enseñanza de la escritura y libros de texto}

Siguiendo lo anteriormente establecido, este estudio parte del reconocimiento de las "huellas de las mediaciones culturales y didácticas" que se inscriben en los materiales. Sobre las huellas de los OA, analizaremos específicamente aquellos trazos vinculados a la didáctica específica. Esta opción por el objeto disciplinar LyL implica una decisión empapada de sentido teórico-metodológico que se fundamenta en la concepción de las disciplinas como un entramado de rituales, hábitos, reglas, modos de formación y acreditación de ciertos grupos y órdenes jerárquicos que, lejos de ser una sumatoria de contenidos, constituyen un producto genuino y forman parte de un sistema educativo y de una cultura escolar particulares (Viñao, 2002; Cuesta, 2012).

Este enfoque situado e histórico de la disciplina nos permitirá ver, sobre el corte sincrónico que supone el análisis de los materiales didácticos actuales del Portal Educ.ar, aquello que ha pervivido y aquello que ha cambiado (Williams, 1982): los sedimentos en los OA, las características que los definen, ya no en relación con un presente ahistórico que coarta la intepretación, sino en virtud de las rupturas y continuidades respecto de sus antecedentes, los libros de texto, y en relación con los nudos problemáticos que han atra-

\footnotetext{
${ }^{4}$ Para llevar adelante esta tarea, asumiremos un enfoque centrado en el diseño, lo cual implica que no nos adentraremos en la investigación del uso que docentes y alumnos hacen de los OA. Entendemos que una investigación volcada al uso de materiales resulta fundamental, ya que la propuesta que plantea el material se completa en su encuentro con un contexto y una comunidad de interpretación que lo dota de sentido a partir de sus pautas y hábitos culturales (Fish, 1998). Al asumir esto, nuestro estudio pretende constituirse en un aporte para una comprensión más profunda de las configuraciones tanto tecnológicas como didáctico-disciplinares que atraviesan los OA, en el camino hacia futuras investigaciones sobre las formas que adopta la construcción de sentido fruto del uso de materiales en contextos concretos.
} 
vesado la enseñanza de LyL en las últimas décadas; entre ellos, particularmente, aquellos vinculados a la enseñanza de la práctica de escritura 5 .

Históricamente, la escritura ha ocupado un lugar central entre las prácticas enseñadas en la escuela (Alvarado, 2001). Sin embargo, en los años 90, momento en que se desarrolla la reforma educativa, esa centralidad toma en la Argentina un cariz nuevo y la escritura adopta características particularmente relevantes para el proceso que pretendemos exponer en torno a los materiales didácticos del área de LyL. En el marco de una progresiva internacionalización y estandarización de la educación, se produjo en este momento una nueva construcción -desarrollada por especialistas formados mayormente en lingüística- en torno a la lectura y la escritura. A partir de la reforma, estas prácticas comenzaron a ser entendidas como habilidades o competencias necesarias para que los alumnos enfrenten las exigencias del mundo actual. En línea con esto, "los Contenidos Básicos Comunes para el área de Lengua y sus fundamentaciones se justificaron en un horizonte utilitarista distinto al de la formación humanística que se resemantizó en la idea de una formación de ciudadanos "competentes". Es decir, que acrediten las competencias necesarias para leer y escribir textos representativos de la complejidad discursiva de la era tecnológica y mediática, mundo globalizado, etc.”(Cuesta, 2012: 45).

Leída desde el diseño disciplinar de los CBC y las políticas educativas de la historia reciente de nuestro país, la enseñanza de la escritura se reconceptualiza. El marco didáctico-disciplinar arroja nueva luz sobre el objeto, que ahora puede ser entendido como parte de la orientación que toma la enseñanza a partir de la reforma educativa de los 90 y que, aunque con modificaciones, se proyecta de forma sostenida en años posteriores. Si bien no continuaremos aquí con la historización de la reforma curricular, ya que no es el objetivo de este trabajo, sí, en cambio, retomaremos los aportes de las investigaciones que exponen el nuevo lugar que ocupa la escritura en los libros de texto de la disciplina de LyL, principalmente, desde la década de los 90 en adelante, y que guarda una estrecha relación con la breve historización que hemos expuesto.

En un estudio dedicado a los libros de texto de la década de 1990, Bombini y Krickeberg (1995) dan cuenta del lugar ganado por el mercado editorial ante la ausencia de reformas de planes de estudios y programas que acompañaran la necesidad de renovación manifestada por diferentes actores del sistema educativo. Antes incluso de la implementación de los CBC, los libros de texto fueron los encargados de incorporar nuevos contenidos que trascendieron los límites de la perspectiva estructuralista imperante hasta ese entonces. Siguiendo esta línea, Bombini señala algunas de las consecuencias de las transposiciones didácticas que toman cuerpo en los libros de texto de ese momento, atravesados por la adopción del llamado enfoque comunicativo: entre otras, el abandono

\footnotetext{
5 La opción por los materiales dedicados a la enseñanza de la escritura -entendida como una alternativa frente al campo de la didáctica de la escritura, que abordan otros estudios- resulta, en definitiva, subsidiaria del recorte realizado en el apartado anterior. Tal como lo entendemos, el abordaje de un objeto como la enseñanza de la escritura no puede explicarse por fuera del enfoque histórico y situado que hemos adoptado de la didáctica de la Lengua y la Literatura, el cual explica los sentidos que esta práctica ha tenido en las últimas décadas en el marco de la escuela.
} 
del texto literario como modelo lingüístico para la escritura y como soporte privilegiado para el trabajo con la lengua; la reabsorción de la literatura en la noción más amplia de discurso y el ingreso a la escuela de diversos géneros discursivos antes relegados, desvinculados de las prácticas de lectura y escritura desarrolladas en la escuela; la introducción de nociones discursivas en relación con la escritura; la superposición de campos de saber provenientes de marcos teóricos diversos, que dan como resultado perspectivas de análisis que terminan por aplicarse a objetos para los cuales no fueron inicialmente pensadas; la utilización puramente descriptiva de las categorías de análisis de la lingüística textual, que se traduce en la pérdida de foco de los objetivos de la escuela: entre ellos, la contribución a la enseñanza de los procesos de comprensión y producción de textos, cuyo descuido había sido, paradójicamente, una de las críticas esgrimidas contra el abordaje del análisis sintáctico de raíz estructuralista que el enfoque comunicativo buscó reemplazar. Paralelamente, encontramos estudios que dan cuenta del alcance que, junto con los aportes del enfoque comunicativo, a partir de la década de los 90 y hasta la actualidad ha tenido el enfoque de la escritura como proceso (Flower \& Hayes, 1996), particularmente, en los libros de texto ${ }^{6}$ (Colafigli, 2011; Finocchio, 2009; Tosi, 2014). El planteo de este enfoque retoma las líneas de la psicología cognitiva para establecer, en grandes líneas, que los procesos que se desenvuelven a la hora de escribir configuran un saber que involucra diversas operaciones por parte de los sujetos y que podríamos resumir muy sintéticamente en términos de planificación, puesta en texto y revisión. A su vez, en el marco de este enfoque, los aportes de la piscología cognitiva se articulan con los planteos de la lingüística textual, a la que hemos hecho referencia con el trabajo de Bombini y Krickeberg (1995). De este modo, la cohesión, la coherencia, las diferentes tramas y tipologías textuales, entre otros, pasan a organizarse en función de los procesos mentales desarrollados por los escritores expertos y se convierten en pautas de escritura para los escritores inexpertos, es decir, los alumnos.

Otro de los enfoques teórico-metodológicos sobre la enseñanza de la escritura que, según diversas investigaciones, irrumpe en la escuela durante los años 90 es la línea del taller de escritura (Bomibini, 2005; Alvarado, 2001; Frugoni, 2006). Desde un lugar no hegemónico y por fuera de los diseños curriculares de la reforma de la década de 1990, el taller se concibe como un espacio donde, a través del trabajo con consignas, se privilegia la invención, el desafío y la experimentación lúdica con el lenguaje. También se trata de una propuesta que busca interpelar los sentidos que atraviesan la escritura en su relación dialógica con la lectura y con la reescritura, y en su vínculo con la escucha y el comentario de los sujetos lectores y escritores propios de la escuela. Si bien existen diversas propuestas editoriales de vanguardia que se han constituido en exponentes puros de esta

\footnotetext{
${ }^{6}$ Este enfoque, que ocupa un lugar significativo entre los lineamientos curriculares tanto de los CBC de Lengua como de los actuales NAP (Ministerio de Educación de la Nación, 2011), profundiza su ingreso en nuestro país durante la reforma, a través de diversas publicaciones de Daniel Cassany (1997). Su injerencia a nivel local ha sido interpretada como producto del movimiento de importación tanto de la reforma española como de su industria editorial, en el marco de los negociados de las editoriales multinacionales (Gerbaudo, 2006).
} 
línea, es posible detectar en varios libros de texto la incorporación de consignas de taller de escritura que se articulan con los ejes de trabajo planteados por el paradigma de la escritura como proceso (Tosi, 2014). Los riesgos de este cruce se visibilizan en aquellas propuestas que transforman la consigna de taller en una serie de pasos secuenciados a los cuales el alumno debe ceñirse para lograr el producto escrito y que coartan el carácter de desafío de invención propio de las consignas de taller.

Por último, más acá en el tiempo, hemos encontrado estudios que señalan que en el marco de la Ley de Educación Nacional y los Núcleos de Aprendizaje de Lengua más recientes, la modalidad de taller de escritura ha pasado a ocupar un lugar fundamental, cuyo impacto se hace visible en los libros de texto del nivel secundario (Tosi, 2014). Allí, las propuestas de taller surgidas en los 80 y llevadas a la escuela en la década de los 90 por referentes como Gloria Pampillo y Maite Alvarado se complejizan con una nueva dimensión vinculada a los aportes de las perspectivas psicogenéticas de enseñanza de la escritura (Lerner, 2001). Desde este marco conceptual, los nuevos diseños hacen particular énfasis en la creación de entornos reales de escritura y se centran en la sociabilización de los textos (Tosi, 2014). De este modo, se busca que la escritura no solo se comparta en el aula a partir del intercambio de opiniones e interpretaciones, sino también que llegue a la comunidad escolar y extra escolar. El taller de escritura se convierte así en una "situación de taller", donde la escritura cumple una función sociocomunicativa de construcción del sentido y donde se prescribe que los alumnos participen sistemáticamente de diversas experiencias comunicativas reales para construir sus subjetividades como lectores y escritores. Se trata una mirada desde la cual la enseñanza, en el marco de esas situaciones reales de comunicación, se da por exposición y contacto del alumno con los textos y por la socialización de estos últimos en clase y fuera de ella, y el docente pasa a ser el acompañante o propiciador de un aprendizaje que los alumnos desarrollan sin otra intervención que la que media naturalmente entre ellos y los textos en situaciones reales de escritura (Cuesta, 2012).

De forma muy sintética, hemos recuperado algunos estudios que analizan los enfoques que configuran las propuestas de enseñanza de la escritura en distintos libros de texto de LyL de diversos períodos de nuestra historia reciente. Estos enfoques no componen un bloque homogéneo en cada momento histórico, ni se suceden linealmente, unos tras otros, a lo largo de las últimas décadas. Por el contrario, en el marco de una historicidad atravesada por procesos políticos, curriculares y editoriales complejos, las investigaciones revelan más bien la existencia de un encastre heterogéneo -aunque no por ello menos historizable- de perspectivas que se cristalizan en los materiales didácticos y conforman un horizonte de rupturas y continuidades invisibles desde un corte puramente sincrónico que descarte la densidad propia de la cultura disciplinar. 


\section{Metodología}

La metodología seleccionada para llevar adelante esta investigación es netamente cualitativa (Goetze \& Lecompte, 1998) y su desarrollo se enmarca en los lineamientos de la Teoría Fundamentada (Strauss \& Corbin, 2002). En primer lugar, se exploró el contexto de los OA, constituido, primero, por el Portal Educ.ar y, luego, por la sección Recursos, donde se encuentra el repositorio de OA. Mediante el uso del buscador destinado a localizar los distintos materiales, se realizó una inmersión inicial en el campo y se relevó un total de 60 OA. Dada la heterogeneidad de este objeto, se optó por realizar un segundo relevamiento y de allí se extrajo una muestra homogénea (Baptista Lucio et al., 2006). Esto quiere decir que, para este primer muestreo, se seleccionaron quince casos de características similares que permitieran reconocer similitudes y hacer un recorte respecto de los restantes OA del Portal.

La selección de estos primeros quince recursos estuvo regida por dos criterios. Primero, se buscaron OA con características similares. Así, se seleccionaron las denominadas Secuencias Didácticas ${ }^{7}$, que presentan características estructurales similares ${ }^{8}$. Segundo, elegimos las SD destinadas a la enseñanza de LyL y, particularmente, a la enseñanza de la escritura.

A fin de llevar adelante la codificación de datos, nos valimos de la Teoría Fundamentada y de su método comparativo constante. En este marco, una vez realizada la exploración del contexto del Portal, el primer relevamiento y la selección de muestras, desarrollamos sucesivas lecturas de las SD extraídas de la sección recursos con el objetivo de descubrir relaciones entre ellas. En el marco de ese proceso, comparamos los datos obtenidos para determinar una denominación común al conjunto de datos que compartían significados similares. La denominación utilizada surgió tanto de la bibliografía de las áreas de Didáctica de la Lengua y Literatura y Tecnología Educativa que sirven de marco teórico para este trabajo, como de las propias propuestas analizadas.

De este modo, comenzamos por enfatizar las similitudes y minimizamos las diferencias a fin de establecer categorías teóricas. Luego, llevamos a cabo el movimiento inverso: remarcamos las diferencias para determinar las propiedades de cada categoría.

\footnotetext{
${ }^{7}$ A partir de ahora, SD.

${ }^{8}$ La denominación que adoptan los OA analizados nos reenvía a un género discursivo del ámbito escolar. Las secuencias didácticas son, en este contexto, series ordenadas de actividades guiadas por un objetivo didáctico global que las dota de sentido. Esta caracterización genérica define de igual forma las SD del Portal Educ.ar, que se encuentran organizadas sobre un espacio que emula una página - proponiendo así una lectura de izquierda a derecha y de arriba a abajo- y distribuyen en partes ordenadas secuencialmente las actividades propuestas para su realización en clase. Siguiendo esta orientación las SD se componen de una serie de etiquetas o metadatos (autor/a; responsable disciplinar; área disciplinar; temática y nivel) y una serie de apartados ordenados en el siguiente orden descendente: Propósitos generales, Introducción a las actividades, Objetivo de las actividades, Objetivos pedagógicos, Enlaces de interés y utilidad para el trabajo y, en último lugar, Bibliografía/Webgrafía recomendada. Si bien en estudios futuros nos dedicaremos a investigar las implicancias de los diversos apartados que componen los OA, aquí nos detendremos particularmente en los Objetivos Pedagógicos, que presentan, dentro de las SD, las consignas de escritura, objeto de nuestro análisis.
} 
En este movimiento, resultó fundamental la bibliografía específica. Esta nos permitió sistematizar las propiedades de las diversas categorías.

Finalmente, nos enfocamos en detectar las relaciones entre las categorías realizando cruces que entendemos son significativos en los campos involucrados en nuestra investigación. Como resultado de este proceso, se apuntó a generar teoría a través de la elaboración inductiva de categorías.

\section{Resultados del análisis}

\subsection{Hipertextualidad y fragmentación}

El análisis de las SD del Portal Educ.ar arroja persistencias y rupturas respecto de las propuestas que hemos detectado en otros materiales didácticos del área de LyL, como son los libros de texto. En primer lugar, hemos reconocido consignas de escritura ${ }^{9}$ cuyo objetivo es la enseñanza de un género o un tipo textual específico. En otros estudios, este tipo de propuestas ha recibido la denominación de Consignas para conocer los géneros: "Se trata de consignas de escritura que, luego de la lectura de textos-modelo y del análisis de las características estructurales, proponen que los alumnos reconozcan a través del escribir unas organizaciones textuales" (Finocchio, 2009: 101). Estas se ubican mayormente sobre el final de las SD y, tal como se desprende del análisis, proponen que los alumnos vuelquen en la escritura los elementos reconocidos o clasificados en las actividades de comprensión lectora, anteriores a la consigna de escritura. Por ejemplo, en una SD dedicada al relato y al texto teatral, luego del planteo de actividades de comprensión lectora, encontramos la siguiente consigna:

"Elegir alguno de los relatos leídos en la actividad 1, y a partir de él escribir un breve texto teatral para ese cuento. Para ello, tener en cuenta las características propias del texto teatral y cómo se presentan los diálogos en ese relato. Para realizar esta actividad pueden utilizar el procesador de textos instalado en los equipos portátiles.” (Educ.ar, 2011)

\footnotetext{
9 Antes de comenzar a abordarlas, vale la pena subrayar que nuestro análisis buscará centrarse en las consignas de escritura de las SD del Portal. Sin embargo, a diferencia de otros estudios, estas consignas no serán analizadas de forma autónoma y autosuficiente, sino más bien de manera integral, como parte de un todo, la SD. Esta decisión metodológica responde a que, tanto en las normativas curriculares recientes (NAP), como en los materiales didácticos del área que conforman los antecedentes de los $\mathrm{OA}$, la escritura, más que un contenido, constituye un eje articulador de contenidos destinado a atravesar la asignatura y a vincular distintos sectores del currículum (Configli, 2011). A su vez, desestimar la relación que las consignas de escritura guardan con otros tipos de consignas destinadas a otros contenidos no solo significaría parcializar y simplificar la función que cumplen en el marco de las secuencias didácticas. También implicaría una operación de invisibilización de las relaciones entre escritura y lengua, literatura y lectura, que han atravesado históricamente la identidad de la enseñanza de esta práctica.
} 
La crítica que se la ha formulado a esta clase de consignas es que parte del supuesto de que el reconocimiento de los componentes y las estructuras de los textos leídos resulta condición suficiente para que los alumnos aprenden a escribir. Resulta fundamental aquí señalar que no negamos que ese saber sea central para el acceso a la escritura, pero por sí solo resulta insuficiente (Finocchio, 2009), ya que, tal como hemos detectado, en este tipo de consignas se quita el foco de otros saberes fundamentales como los que configuran la dimensión discursiva de los géneros abordados ${ }^{10}$. Se trata de una operación de reificación a partir de la cual la naturaleza discursiva de los géneros, sus circuitos de producción y circulación, su historicidad y su carácter multimediático no son tomados en consideración en el marco de las actividades de comprensión lectora; omisión que luego se traslada a las consignas de escritura, que incorporan únicamente las características de las tipologías textuales como parte de sus desafíos.

Nos interesa en este punto, preguntarnos cómo se articula la hipertextualidad de las SD con las líneas didáctico-disciplinares detectadas en los materiales. Cuando repasamos la literatura dedicada a analizar el fenómeno hipertextual, vemos que la fragmentariedad es una de las características más frecuentemente aludidas. Es así que, de forma muy similar a otros teóricos vinculados al posestructuralismo, George Landow (1995) caracteriza al hipertexto esencialmente como un texto compuesto de fragmentos de textos conectados entre nexos electrónicos. Siguiendo esta línea, diversos estudios entienden que como consecuencia de la fragmentariedad del hipertexto, los lectores han ganado libertad para navegar y leer según sus propios criterios, al margen de las secuencias lineales impuestas por los textos impresos, no hipertextuales, y de este modo, se confunden con los autores de los textos que leen (Landow, 1995; Burbules y Callister, 2001).

Como respuesta a este enfoque, retomamos el planteo de Piscitelli (2004), quien luego de establecer que hay diferencias entre elegir en qué dirección se navega y ser el autor de un texto, afirma que aquellos que, como Landow, apoyan la indistinción entre lectores y autores confunden la epistemología propia de la recepción de un texto y la estructura que organiza los hipertextos. En esta misma línea se ubican los estudios sobre materiales didácticos en entornos virtuales (Gergich et al., 2012) al plantear en sus desarrollos que la linealidad y la tabularidad están presentes en textos e hipertextos, y que un material didáctico puede ser de naturaleza secuencial por estar distribuido en clases u organizar jerárquicamente sus títulos, al margen de que posea un alto número de hipervínculos.

Este es el caso también de las SD que presentan Consignas para conocer los géneros, donde la incorporación de hipertextos no decanta en una ruptura de la secuencialidad de la lectura ni en una supuesta libertad del lector para elegir su camino entre los hipertextos pautados por el material. Por el contrario, como revela nuestro análisis, en

10 Bombini y Krickeberg (1995) conceptualizan este problema en torno a los libros de texto en el período de los CBC. El ingreso del enfoque comunicativo en las propuestas editoriales de ese momento trajo, entre otras dificultades, la incorporación de "un género pero no una perspectiva de análisis apropiada al mismo. Así en uno de los libros de Lengua y Literatura los guiones de "Mafalda" de Quino son propuestos para explicar la clasificación de las oraciones según la actitud del hablante, los signos de entonación y el predicado verbal y no verbal.” (p. 26). 
estas SD los hipertextos se organizan a partir de la linealidad que propone el género discursivo escolar. Este involucra, además de un tono predominantemente instructivo, un orden de actividades secuenciadas, por lo que las consignas de lectura que se proponen desde la secuencia suponen un orden determinado, y la organización de los textos enlazados por los hipertextos se torna dependiente de esa propuesta integral.

Ahora bien, esto no quiere decir que no exista relación alguna entre la hipertextualidad de los OA y la fragmentariedad que atraviesa las SD que presentan Consignas para conocer los géneros. En este tipo de propuestas, abundan los hipertextos que enlazan las páginas de las SD con sitios del Portal Educ.ar diseñados única y específicamente para albergar breves fragmentos de relatos, cuentos y obras de teatro. Estos pequeños fragmentos suelen carecer de una contextualización que permita ubicar su sentido en la obra que les sirve de marco. En la mayoría de los casos, tampoco puede reconocerse una referencia bibliográfica, ya sea textual o hipertextual, que nos referencie a otros sitios donde se encuentre la obra completa.

Siguiendo esta misma línea, el planteo de las consignas de lectura que involucran estos hipertextos tampoco ofrece indicación alguna en cuanto a las dimensiones discursivas de la obra en el sentido al que hemos hecho referencia en apartados anteriores:

Les proponemos que lean con sus alumnos algunos retratos $(\underline{1}, \underline{2}, \underline{3}, \underline{4})$ tomados de diferentes textos literarios. Al finalizar, ellos pueden realizar las siguientes actividades: / ¿Qué personaje es retratado? Investigar en enciclopedias o en internet sobre ellos. / ¿Cuáles de ellos fueron personajes históricos y cuáles fueron creaciones literarias? / Indicar qué aspectos del personaje están presentes en cada retrato: -partes del cuerpo -manera de vestir-sentimientos -modo de pensar -hábitos -personalidad -edad. (Educ.ar, 2011a)

En estos casos, más que una propiedad del diseño hipertextual, la fragmentación de las SD surge de la operación de recorte y descontextualización a las que se ven sometidos los textos a través del uso de hipertextos (en este caso, en forma de números). Esta operación no es inmotivada y puede explicarse a partir de la conceptualización realizada por Bombini y Krickeberg (1995) en lo referido a las consecuencias del llamado enfoque comunicativo y a la tergiversación de marcos teóricos aplicados a objetos para los cuales no fueron originalmente ideados. Si en las SD, como hemos detectado en nuestro análisis, hay una fragmentación de las obras de teatro, los retratos o los relatos es porque allí se entiende que para analizar, por ejemplo, los diálogos, el tema, los personajes, la trama narrativa o descriptiva de un texto, resulta prescindible abordar la obra de teatro, el retrato o el relato en tanto tales. Desde la propuesta del enfoque comunicativo, la literatura es entendida como un tipo textual entre otros tipos textuales, y, por tanto, leer hipertextos se transforma en una operación de lectura de tipos textuales o géneros discursivos en un sentido acotado, para cuyo análisis el breve recorte descontextualizado de una obra es funcional. 
Desde este enfoque, entonces, la fragmentación se reconceptualiza, ya no como una ruptura de la lectura secuencial o como la puerta de entrada a una lectura descentrada y libre, sino más bien como una propuesta didáctica de lectura que continúa ciertas líneas plausibles de ser rastreadas en los materiales de LyL de nuestra historia reciente, y que tiene una incidencia directa en los desafíos de las consignas de escritura de los materiales.

\subsection{Hipermodalidady fragmentación}

En el caso de las SD que incorporan las posibilidades hipermodales ${ }^{11}$ que brinda la Web 2.0, la omisión de la dimensión discursiva se articula con otra omisión, vinculada al carácter multimodal de los objetos culturales del soporte digital. Es el caso, por ejemplo, de los OA que enlazan videos a sus consignas. Veamos el caso de la SD titulada "Las características del género de ciencia ficción":

"Si cuentan con conexión en el colegio, los invitamos a comenzar las actividades mirando junto con sus alumnos algunos de estos tráilers de películas de ciencia ficción (u otros que encuentren en internet). 1. Yo, robot / 2. La guerra de los

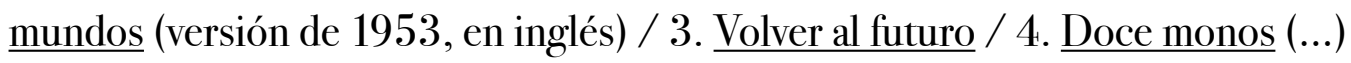
Luego, realice la siguiente consigna: ¿Qué elementos de estas películas hacen que pertenezcan al género ciencia ficción?” (Educ.ar, 2011b)

El desarrollo posterior a esta propuesta de visualización apunta principalmente a que los alumnos clasifiquen los tráilers según distintas temáticas propias del género de ciencia ficción provistas por la SD (ecología, explosión-guerra nuclear, explosión demográfica, plagas, entre otras) y comparen las características del género en cuestión con otros géneros, como el fantástico. Esta clasificación temático-genérica propia de los abordajes textuales del enfoque comunicativo no indaga en el carácter multimodal de los tráilers en tanto objetos culturales, sino que, mediante la categoría de género borra su diferencia respecto de otros objetos culturales. Esto se ve claramente en el momento en que, más adelante en la misma SD se aborda el género ciencia ficción, pero ahora, a partir de la lectura de cuentos. Leemos en una pregunta diseñada para trabajar el cuento "El cosmonauta”, de Ángel Arango: “Bajo qué temática clasificarían este cuento?”

\footnotetext{
${ }^{11}$ La hipermodalidad (Lemke, 2002) resulta de la articulación de la hipertextualidad, que hasta aquí hemos abordado en nuestro análisis, y la multimodalidad, sobre la cual hacemos una breve referencia: las investigaciones de la semiótica multimodal parten de la base de que la construcción de sentido que se desarrolla en las esferas de la vida humana excede la palabra. Si bien esto no es algo novedoso, porque los modos -visual, audiovisual, táctil, entre otros-, entendidos como recurso social y culturalmente modelados para construir significados (Kress, 2009: 54), siempre han existido, también es cierto que los objetos que hoy pueblan los entornos digitales permiten integrar de forma aún más dinámica esos diferentes modos. En este marco, la hegemonía de la palabra es puesta en suspenso para abrir el análisis a las formas en que los modos conviven de forma articulada.
} 
Cuentos y tráilers son objeto de una misma operación didáctica: la propuesta de atribución de algunas propiedades específicas, genéricas, y el descarte de otras características que reconcilian al tráiler con su circulación, su producción, su función social, su ideología y, por último, como hemos agregado en este apartado, con las características multimodales que definen las formas de construir sentido de los objetos culturales por fuera del ámbito de la palabra entendida de modo aislado.

Si continuamos con el análisis de este ejemplo, vemos que luego de las consignas de reconocimiento de temas, personajes, marcos espacio-temporales de los cuentos, se selecciona uno de ellos, "Últimas imágenes desde Buenos Aires”, para llevar adelante la consigna de escritura:

"Discutir con sus compañeros de grupo cómo se desarrollaría una película basada en «Últimas imágenes desde Buenos Aires». Redactar el guión del tráiler (seleccionando las partes que pueden resultar más atractivas para el público, pero evitando revelar el final). Por último, filmarlo.” (Educ.ar, 2011b)

Este movimiento que va de las consignas de comprensión del tráiler sobre el inicio de la SD, pasando por las de comprensión lectora de los cuentos, hasta la culminación de la SD con la consigna de escritura recién citada nos permite comprender algunas de las características que pueden adoptar tanto la hipertextualidad como la hipermodalidad cuando se incorporan a las denominadas Consignas para aprender los géneros. La omisión de variables discursivas y multimodales se sintetiza en la naturaleza de las vallas o desafíos que conforman la consigna. Para escribir un guión no hay más que tener en cuenta las consideraciones temáticas realizadas al principio de las SD, sumadas a las dos características del género tráiler que se agregan en la consigna de escritura: incorporar "las partes que pueden resultar más atractivas para el público, pero evitando revelar el final". Nuevamente, la consigna de escritura resulta posible gracias a una concepción reduccionista -vehiculizada, esta vez, por los enlaces hipermodales- que privilegia las categorías textuales y genéricas como únicos conocimientos necesarios para desarrollar la práctica de escritura en el marco de las consignas.

\subsection{Hipermodalidad y quiebre}

Por otro parte, hemos detectado que también existen SD en el marco de las cuales el uso de la hipermodalidad representa un quiebre y no una continuidad respecto de las problemáticas que hasta aquí hemos planteado. Son casos en los que, en lugar de ser descartado, el carácter multimodal de las producciones audiovisuales habilita un abordaje alternativo. Observemos el ejemplo que nos ofrece la SD “Trama textual instructiva, oral y escrita” en su primera actividad. Allí se propone la visualización de un video del programa "Cocineros argentinos" donde se explica cómo realizar un tipo particular de torta frita: 
Les proponemos que junto con sus con sus alumnos vean un video con una receta muy original: la torta frita del embudo. / ¿Qué acciones del cocinero Juan acompañan la explicación oral? / ¿Qué pasos recuerdan que debemos seguir para hacer una torta frita del embudo? / ¿Con qué objetivo la gente mira un programa de cocina, de manualidades, etcétera? (Educ.ar, 2011c)

Aquí podemos ver cómo, en lugar de avanzar sobre los rasgos de la trama instructiva o sobre aquellas características que presentan por igual las recetas de los programas televisivos y los libros de cocina, de modo similar al ejemplo del apartado anterior, aquí, en cambio, las preguntas que acompañan al video plantean un indagación vinculada a la construcción de sentido desde lo visual y oral -las acciones del cocinero del programa- $y$ al consumo cultural de este tipo de programas -los objetivos de los televidentes-.

Este que hemos analizado no es un ejemplo aislado. Más adelante, luego de proponer la lectura de una receta escrita, la misma SD plantea la siguiente consigna:

Comparar la receta escrita con la que prepararon en vivo en «Cocineros Argentinos». ¿Cómo nos indica cada una la forma de preparar una torta frita del embudo? ¿Alguna de las dos lo indica de manera más eficaz? ¿Por qué? ¿Cómo lo hace? (Educ.ar, 2011c)

Al proponer una operación de comparación entre la receta escrita y la receta audiovisual, la consigna que hemos citado apunta a desencadenar una distinción y una conceptualización de los lenguajes o modos que las atraviesan. Esta propuesta de comparación de modos o lenguajes se alinea con las indagaciones de los estudios sobre multimodalidad $^{12}$, que, trascendiendo los análisis puramente textuales, buscan comprender tanto las combinaciones entre los distintos modos como las posibilidades de los modos para construir sentido. Tal como establece Magadán, (2014: 5): "Por su materialidad, cada modo tiene diferentes potenciales -y también limitaciones- para construir sentido, y esos diferentes potenciales determinan qué modo resulta conveniente seleccionar según cada situación de comunicación específica".

Ahora bien, resulta llamativo que cuando nos adentramos en el análisis de la consigna de escritura sobre el final de la SD, la línea de la multimodalidad no es retomada. El diálogo posible entre escritura y producción audiovisual, y la profundización de la línea que apunta a indagar la naturaleza discursiva de las recetas se ven interrumpidos. Leamos la consigna que nos ofrece la SD en su parte final:

\footnotetext{
12 Tal como explica Cecilia Magadán, este tipo de estudios asumen que los objetos de lectura y escritura mediáticos y digitales están hechos de algo más que palabras: "páginas y sitios web, blogs, wikis, videojuegos, libros digitales combinan diferentes lenguajes o modos y, por lo tanto, se conciben en el marco de la multimodalidad” (Magadán, 2014: 5).
} 
Escribir un texto breve (entre quince y veinte renglones) en el que predomine la trama textual instructiva. Elegir entre las siguientes propuestas u otra del mismo estilo que se les ocurra: / -Instrucciones para reír. / - Instrucciones para comer fideos con tuco sin mancharse. / - Instrucciones para meterse en el agua cuando está muy fría. / - Instrucciones para conquistar a una chica o a un chico. (Educ. ar, 2011c)

La orientación de esta consigna de escritura se explica mejor cuando retomamos su contexto de aparición en la SD. Antes de esta consigna, se presentan una serie de actividades de comprensión lectora. Estas, a su vez, se encuentran luego de las consignas vinculadas a la multimodalidad que hemos analizado. En las actividades de comprensión lectora, la propuesta de análisis de tres cuentos distintos se reenmarca en la orientación fragmentaria del enfoque comunicativo con consignas del tipo: “¿A qué género discursivo pertenece este texto: narrativo, lírico o dramático? ¿Por qué? ¿Qué recursos utiliza?” "Marcar ejemplos de las figuras retóricas que aparecen (paralelismo, anáfora, hipérbole, comparaciones, antítesis, etcétera)." Tal como lo entendemos, la consigna de escritura retoma esta orientación comunicativa de las actividades de comprensión lectora que la anteceden y, de este modo, se deshace de la ruptura que supone el ingreso de la reflexión en torno a la multimodalidad. No es casual, visto desde esta perspectiva, que la incorporación de hipertextos que enlazan videos se desarrolle en las primeras partes de las secuencias didácticas, a distancia de las consignas de escritura. Tampoco lo es que entre estas últimas y los objetos multimodales medie, como hemos visto en diversas SD, el abordaje de textos propio del aparato de análisis del enfoque comunicativo.

\section{Conclusión}

A lo largo de este trabajo, hemos perseguido el objetivo de explorar los aportes que la didáctica de LyL puede realizarle a los estudios dedicados a comprender la inclusión de tecnologías digitales en materiales didácticos. En este sentido, a partir del análisis de algunos ejemplos representativos de una muestra más amplia, hemos intentado lograr un mayor grado de inteligibilidad en el análisis del diálogo que se establece entre las posibilidades que ofrecen fenómenos vinculados a la hipertextualidad, multimodalidad e hipermodalidad, y la enseñanza de la escritura, entendida como un práctica que se desarrolla en el marco de una disciplina específica.

El estudio de las SD arroja casos en que las posibilidades ofrecidas por las tecnologías digitales se pliegan, como una continuidad, a enfoques problemáticos de la enseñanza de LyL que pueden rastrease en la reforma de la década de 1990. Tal es el caso de las SD que presentan Consignas para aprender los géneros. Allí, las producciones audiovisuales enlazadas hipermodalmente por las SD se ven reducidas a una operación de com- 
prensión fragmentaria, similar a la detectada en las actividades de lectura de hipertextos. En estos casos, las características textuales y genéricas señalizadas en las producciones audiovisuales y en los textos pasan a formar parte de los desafíos que involucran las consignas de escritura.

También detectamos casos en que las producciones audiovisuales son abordadas a partir de consignas de comprensión que contemplan sus dimensiones multimodales y discursivas. Sin embargo, esta línea de reflexión no se continúa en las consignas de escritura de esas SD. Al igual que en los casos antes expuestos, las Consignas para aprender los géneros terminan por descartar la dimensión multimodal de las producciones audiovisuales, para proponer, en cambio, desafíos que fragmentan y reifican la práctica de escritura en su dimensión discursiva.

Antes de concluir, resulta fundamental señalar el punto de contacto que existe entre los estudios críticos de este tipo de abordajes de la enseñanza (Bombini \& Cuesta, 2006; Cuesta, 2012) y las investigaciones provenientes de la línea de la semiótica multimodal (Kress, 2009, Cope \& Kalantzis, 2012) volcada a las tecnologías digitales. A pesar de la distancia que hay entre sus objetos de estudio, tanto una como otra línea coinciden, primero, en señalar la necesidad de realizar una apertura de los discursos -en el caso de la primera- y modos -en el caso de la segunda- trabajados en el ámbito escolar.

La propuesta realizada desde la didáctica de la LyL apunta a dar cuenta de todo el discurso social a fin de desclausurar los campos autónomos que conforman la literatura, la filosofía y la historia, entre otros, $\mathrm{y}$, de este modo, abordar todo aquello que se dice y escribe en un estado de sociedad; lo impreso, lo hablado, lo representado en los medios electrónicos. Desde los estudios sobre la multimodalidad se parte de la crítica al énfasis privativo que la escuela ha puesto históricamente en la enseñanza de la palabra y de lo escrito, desligándose de los otros modos y lenguajes mediante los cuales se construye sentido en la actualidad.

Segundo, ambas líneas plantean la necesidad de un trabajo sistemático sobre la diversidad que significa esta apertura. En el caso de la didáctica, si bien, como hemos dicho, su propuesta apunta a una consideración más amplia del discurso social, esto no significa realizar un abordaje de la totalidad del discurso empírico, que resultaría redundante, sino que, más bien, implica conceptualizar la reglas que rigen los enunciados en un contexto social determinado, los sistemas genéricos, los repertorios tópicos que se reiteran y que organizan lo decible en una sociedad, y las hegemonías y contrahegemonías que atraviesan el discurso. Por su parte, los estudios que se enmarcan en el paradigma de las nuevas alfabetizaciones proponen una gramática de la multimodalidad para la escuela que permita estudiar sistemáticamente las formas de construir sentido posibilitadas por cada modo y por las combinaciones de distintos modos en las producciones culturales que atraviesan nuestra cultura. 


\section{Bibliografía}

- Alvarado, M. (2001). Enfoques en la enseñanza de la escritura. En Entre líneas. Teorías y enfoques en la enseñanza de la escritura, la gramática y la literatura (13-51). Bs. As.: Flacso/Manantial.

- Alvarado, M., \& Bombini, G. (1995). Elnuevo escriturón. Buenos Aires: El Hacedor.

- Área Moreira, M. (2004). Los medios y las tecnologías en la educación. Madrid: Pirámide.

- Baptista Lucio, P.; Fernández Collado, C.; Hernández Sampieri, R. (2006). Metodología de la Investigación. México DF: Editorial McGraw-Hill Interamericana.

- Bombini, G. (2006). Reinventar la enseñanza de la Lengua y la Literatura. Buenos Aires: Libros del Zorzal.

- Bombini, G., \& Cuesta, C. (2006). Lengua y Literatura: campo de la didáctica específica y prácticas de enseñanza. En Fioriti, Gema (comp.), Didácticas Específicas. Reflexiones y aportes para la enseñanza (53-77). Buenos Aires: Miño y Dávila.

- Bombini, G. \& Krickeberg, G. (1995). De texto los libros I. En Bombini, G., Otras tramas. Sobre la enseñanza de la lengua y la literatura (pp. 23-43). Rosario: Homo Sapiens.

- Bomibini, G. (2001). La literatura en la escuela. En Alvarado, M. [coord.]. Entre líneas. Teorías y enfoques en la enseñanza de la escritura, la gramática y la literatura (53-74). Buenos Aires: Manantial.

- Burbules, N. \& Callister, T. Jr. (2001). Educación: Riesgos y promesas de las nuevas tecnologías. Buenos Aires: Granica.

- Cassany, D. (1997). Describir el escribir. Cómo se aprende a escribir. Barcelona: Paidós.

- Colafigli, L. (2011). Enseñanza de la escritura. Un análisis de las propuestas en manuales de lengua. Tesis Doctoral.

- Cope, B \& Kalantzis, M. (2012). Literacies. New York: Cambridge University Press.

- Cuesta, C. (2012). Lengua y Literatura: Disciplina escolar. Hacia una metodología circunstanciada de su enseñanza. Tesis doctoral, Facultad de Humanidades y Ciencias de la Educación, Universidad de La Plata.

- De Angelis, B., Gergich, M. \& Imperatore, A. (2010). Materiales didácticos en construcción: una historia posible frente a los desafíos de la Web 2.0. En Pérez Tornero, J. M. (Coord.), Alfabetización mediática y culturas digitales. Universidad de Sevilla. 
- Dussel, I. (2011). Aprender y enseñar en la cultura digital. VII Foro Latinoamericano de Educación TIC y Educación: experiencias y aplicaciones en el aula Documento Básico. Buenos Aires: Santillana.

- Finocchio, A. M. (2009). Conquistar la escritura: saberes y prácticas escolares. Buenos Aires: Paidós.

- Fish, S. (1998) [1987], ¿Hay un texto en esta clase? En Palti, E. J., Giro lingüústico e historia intelectual, Bernal, Universidad Nacional de Quilmes.

- Flower, L., \& Hayes, J. (1996). Textos en contexto. Los procesos de lectura y escritura. Buenos Aires: Lectura y vida.

- Frugoni, S. (2006). Imaginación y escritura. La enseñanza de la escritura en la escuela. Buenos Aires: Libros del Zorzal.

- Gerbaudo, A. (2006). Ni dioses ni bichos. Profesores de literatura, curriculum y mercado. Santa Fe: Centro de Publicaciones de la Univ. del Litoral.

- Gergich, M., Imperatore, A. \& Schneider, D. (2010). Hacia una redefinición del hipermedia educativo en los tiempos de la web 2.0.. En VCongreso de Tecnología en Educación y Educación en Tecnología, Quilmes, Argentina.

- Goetz, J. P., \& LeCompte, M. D. (1988). Etnografía y diseño cualitativo en investigación educativa. Madrid: Morata.

- Grafein (1981). Teoría y práctica de un taller de escritura. Madrid: Altalena.

- Imperatore, A. (2009). Cambios de concepción y usos acerca de los materiales didácticos para la educación superior en entornos virtuales. En Pérez, S. e Imperatore, A. (Comps.), Comunicación y educación en entornos virtuales de aprendizaje: Perspectivas teórico-metodológicas (344-351). Bernal: Universidad Nacional de Quilmes Editorial.

- Kress, G. (2009). What is a mode? En C. Jewitt (Ed.), The Routledge handbook of multimodal analysis. New York: Routledge, pp. 54-67.

- Landow, G. (2009). Hipertexto 3.0. Teoría crítica y nuevos medios en la era de la globalización. Barcelona: Paidós.

- Lerner, D. (2001). Leer y escribir en la escuela: lo real, lo posible y lo necesario. México, FCE. 
- Maggio, M., Lion, C., \& Sarlé, P. (2012). Creaciones, experiencias y horizontes inspiradores. La trama de Conectar Igualdad. Buenos Aires: Educ.ar SE

- Ministerio de Cultura y Educación de la Nación, Consejo Federal de Cultura y Educación (1995). Contenidos Básicos Comunes para la Educación General Básica. 1. a Edición.

Ministerio de Educación, Ciencia y Tecnología de la Nación (2006). Núcleos de Aprendizaje. Lengua tercer ciclo EGB, nivel medio.

- Piscitelli, A. (2004). Escritura no secuencial. Ejemplos. Teoría. Recuperado el 20 de septiembre de 2014 de http://www.ilhn.com/datos/teoricos/archives/001809.php.

- Prendes Espinosa, M. P., F. Martínez Sánchez e I. Gutiérrez Porlán (2008), “Producción de material didáctico: los objetos de aprendizaje", Revista Iberoamericana de Educación a Distancia, 11 (1), 81-10. Recuperado el 20 de septiembre de 2015 de http://www.redalyc. org/articulo.oa?id=331427208004

- Sabulsky, G. (2009). Materiales educativos que recuperen el hacer y el pensar del profesor. En Pérez, S. e Imperatore, A. (Comps.), Comunicación y educación en entornos virtuales de aprendizaje: Perspectivas teórico-metodológicas (344-351). Bernal: Universidad Nacional de Quilmes Editorial.

- Strauss, A \& Corbin, J. (2002). Bases de la investigación Cualitativa. Técnicas y procedimientos para desarrollar la teoría fundamentada. Bogotá: CONTUS.

- Tosi, C. (2014). Representaciones sobre la escritura y su enseñanza en propuestas editoriales para el secundario. Un recorrido entre 1960 y 2010.Traslaciones, 1(2). Recuperado el 20 de septiembre de 2015 de http://revistas.uncu.edu.ar/ojs/index.php/traslaciones/ article/view/244

- Viñao, A. (2002). Sistemas educativos, culturas escolares y reformas: continuidades y cambios. México: Ediciones Morata.

- Williams, R. (1982). Cultura. Sociología de la comunicación y del arte. Barcelona: Paidós.

- Educ.ar (2011). Del relato al texto teatral. Recuperado el 10 de septiembre de 2015 de http://www.educ.ar/sitios/educar/recursos/ver?id=14816\&referente=docentes

- Educ.ar (2011a). El retrato. Recuperado el 10 de septiembre de 2015 de http://www. educ.ar/sitios/educar/recursos/ver?id=14835

- Educ.ar (2011b). Las características del género de ciencia ficción. Recuperado el 10 de septiembre de 2015 de http://www.educ.ar/sitios/educar/recursos/ver?id=14811

- Educ.ar (2011c). Trama textual instructiva, oral y escrita. Recuperado el 10 de septiembre de 2015 de http://www.educ.ar/sitios/educar/recursos/ver?id=14915 